\title{
Radio-frequency magnetron co-sputtered Ge-Sb-Te phase change thin films
}

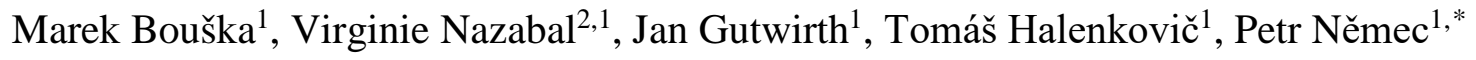

${ }^{1}$ Department of Graphic Arts and Photophysics, Faculty of Chemical Technology, University of Pardubice, Studentská 573, 53210 Pardubice, Czech Republic

${ }^{2}$ Equipe Verres et Céramiques, UMR-CNRS 6226, Sciences Chimiques de Rennes (SCR), Université de Rennes 1, 35042 Rennes Cedex, France

${ }^{*}$ Correspondence and requests for materials should be addressed to P.N. (petr.nemec@upce.cz) 


\begin{abstract}
Radio-frequency magnetron co-sputtering technique with GeTe and $\mathrm{Sb}_{2} \mathrm{Te}_{3}$ targets has been used for the deposition of Ge-Sb-Te amorphous thin films. Fabricated layers cover broad region of chemical composition ( 17.4-35.0 at. \% of Ge, $14.2-29.0$ at. \% of Sb) with slight variation in Te content (50.8-53.6 at. \% of Te). Upon annealing-induced crystallization, large variations in electrical contrast up to eight orders of magnitude were found. Phase change from amorphous to crystalline state leads also to drastic changes of optical functions demonstrated by optical contrast values up to $|\Delta n|+|\Delta k|=2.96$ for GeTe layers at Blu-ray wavelength. Reflectivity contrast at Blu-ray wavelength reaches up to $\sim 43 \%$ with increasing content of GeTe in Ge-Sb-Te thin films, confirming importance of GeTe content in Ge-Sb-Te thin films.
\end{abstract}

Keywords: radio-frequency magnetron co-sputtering; thin films; chalcogenides; phase transitions 


\section{Introduction}

More than 50 years ago, reversible electrical swiching phenomenon in amorphous chalcogenides was first time reported by Ovshinsky [1]. In the end of 1980s and beginning of 1990s, phase change materials based on Ge-Sb-Te [2] and/or Ag-In-Sb-Te [3] systems have been discovered. The main scientific as well as technological interest of these inorganic materials is their ability to transform quickly and reversibly between amorphous and crystalline phases. Fast phase transformation can be induced reversibly through varying the electric field or temperature by heating via a laser pulse in optical recording applications [4, 5]. The extraordinary properties of phase change materials based on Ge-Sb-Te ternary system are connected with changes of optical reflectivity (up to 30\%) and/or electrical resistivity (several orders of magnitude) taking place upon phase transition [6].

The optimization of phase change materials has led to the development of optical storage media such as digital versatile disks (DVD) or Blu-ray disks (BD) [7, 8]. A typical material composition from Ge-Sb-Te system used in applications is $\mathrm{Ge}_{2} \mathrm{Sb}_{2} \mathrm{Te}_{5}$, where the ratio (GeTe):( $\left.\mathrm{Sb}_{2} \mathrm{Te}_{3}\right)$ is 2:1, or $\mathrm{Ge}_{8} \mathrm{Sb}_{2} \mathrm{Te}_{11}$, where $(\mathrm{GeTe}):\left(\mathrm{Sb}_{2} \mathrm{Te}_{3}\right)=8: 1$ [8-10]. Moreover, phase change materials are considered for non-volatile memories and recently for other photonic applications such as integrated all-photonic non-volatile memories, optical color rendering, nanopixel displays, and reconfigurable nanoplasmonic devices or active terahertz photonics $[9,11]$.

For the fabrication of Ge-Sb-Te thin films, different deposition techniques such as thermal (flash) evaporation [12, 13], pulsed laser deposition [14, 15], chemical vapor deposition using metal-organic precursors [16, 17] or atomic layer deposition [18] can be exploited. Another, widely used deposition technique is magnetron sputtering which is considered as a method preserving well the stoichiometry of the target material during the deposition process and producing thin films with good adherence as well as good 
homogeneity and uniformity for industrial processes [19, 20]. Radio-frequency (RF) cosputtering technique brings specifically advantage of deposition thin films with various compositions by only adjusting electrical power ratio applied on individual cathodes, making this method cost-effective for compositional studies of materials’ properties [21]. Moreover, co-sputtering is efficient to fabricate amorphous thin films whose composition is out of the glass-forming region of the system under study [22].

Magnetron sputtering is widely used for the growth of Ge-Sb-Te thin films, as reported for example in Refs [23-25]. However, co-sputtering method is less frequent and mainly used for the doping of Ge-Sb-Te materials with other elements such as C (RF sputtering [26]), Al (RF [26]), Ti (DC [27]), Ni (DC/RF [28]), Cu (DC/RF [29]), Se (DC [30]), Zr (DC/RF [31]), Sn (RF [32]) or Bi (DC [26], RF [32]).

Contrary, RF co-sputtering is used in this work to explore fabrication of thin Ge-Sb-Te films within broad region of chemical composition varying only the electrical power ratio applied to GeTe and $\mathrm{Sb}_{2} \mathrm{Te}_{3}$ sputtering targets without necessity of exploiting many different compositions of the targets from Ge-Sb-Te system when simple RF sputtering is employed.

Thus, in this paper, Ge-Sb-Te thin films with different (GeTe):( $\left.\mathrm{Sb}_{2} \mathrm{Te}_{3}\right)$ ratio were prepared by RF co-sputtering. Fabricated films were characterized in as-deposited state (amorphous phase) as well as in crystalline state (after by thermal annealing) using atomic force microscopy (AFM), scanning electron microscopy (SEM) with energy-dispersive X-ray analysis (EDS), X-ray diffraction (XRD), electrical resistivity, and variable angle spectroscopic ellipsometry data.

\section{Materials and Methods}


The RF magnetron co-sputtering of Ge-Sb-Te thin films was performed at a room temperature using MPE600 multi-chamber deposition system (Plassys-Bestek) equipped with symmetrically arranged confocal deposition cluster consisting of three cathodes [21, 33].

The thin films were fabricated using the 2"’ polycrystalline chalcogenide targets with nominal composition of GeTe and $\mathrm{Sb}_{2} \mathrm{Te}_{3}$ (99.999 \%, ALB Materials, Inc., USA). Thin films were deposited onto borosilicate glass (used for XRD, spectroscopic ellipsometry, and electrical resistivity measurements) and single crystalline silicon wafers $<100>$ substrates (exploited for SEM, EDS, and AFM experiments). The experimental conditions were kept constant throughout all the depositions, i.e. background pressure of $\leq 5 \times 10^{-7} \mathrm{mbar}$, argon working pressure of $5 \times 10^{-3}$ mbar maintained by $75 \mathrm{sscm}$ flow rate and substrate-target distance of about $8 \mathrm{~cm}$ with substrate holder rotation. To study the effect of crystallization, asdeposited films were annealed in inert atmosphere (pure argon) at the temperature of $230{ }^{\circ} \mathrm{C}$ (to assure that the crystallization to fcc structure proceeded) for $120 \mathrm{~min}$ and slowly cooled down to room temperature.

The surface morphology of the Ge-Sb-Te films and their chemical composition were studied using scanning electron microscopy (SEM, JEOL JSM 6400) linked with energydispersive x-ray spectroscopy analyzer. Atomic force microscopy (Solver NEXT, NT-MDT) was used to study topography of Ge-Sb-Te layers within typical scanned area of $5 \mu \mathrm{m}$ x $5 \mu \mathrm{m}$ in a semi-contact mode. X-ray diffraction technique (D8-Advance diffractometer, Bruker AXS) was exploited to determine the structure of Ge-Sb-Te samples using Bragg-Brentano $\theta-\theta$ geometry with $\mathrm{CuK} \alpha$ radiation and secondary graphite monochromator. The diffraction angles were measured at room temperature from 5 to $65^{\circ}(2 \theta)$ within $0.02^{\circ}$ steps. Sheet resistance temperature dependences measurements at $2{ }^{\circ} \mathrm{C} \cdot \mathrm{min}^{-1}$ were carried out using a four-point probe based on van der Pauw method [34]. 
Two variable angle spectroscopic ellipsometers (J. A. Woollam Co., Inc., Lincoln, NE, USA) were employed for the optical characterization of studied materials: first ellipsometer with automatic rotating analyzer for the spectral range 0.3 - $2.3 \mu \mathrm{m}$ (UV-NIR), measuring 100 revolutions with wavelengths steps of $20 \mathrm{~nm}$ at selected angles of incidence (50, $60^{\circ}$, and $70^{\circ}$ ); second ellipsometer with rotating compensator for 1.7-20 $\mu \mathrm{m}$ range using angles of incidence as above, 50 scans, 15 spectra per revolution, resolution of $16 \mathrm{~cm}^{-1}$. For the analysis of VASE data in broad measured spectral region (300 nm - $20 \mu \mathrm{m}$ ), we used Cody-Lorentz (CL) oscillator model which includes the correct band edge function, weak Urbach absorption tail description as well as Lorentz oscillator function [35]; this model is convenient for the description of amorphous chalcogenides optical functions [20, 36]. Drude-type contribution for free carriers was added to the CL oscillator for crystallized samples as well as for asdeposited $\mathrm{Sb}_{2} \mathrm{Te}_{3}$ which is partially crystalline.

\section{Results and discussion}

The chemical composition of deposited Ge-Sb-Te films estimated by EDS is visualized in the ternary diagram (Fig. 1) as well as in Table 1. For a reader's convenience, Fig. 1 shows also selected stoichiometric compositions located on $\mathrm{GeTe}_{-} \mathrm{Sb}_{2} \mathrm{Te}_{3}$ tie-line: $\mathrm{Ge}_{1} \mathrm{Sb}_{2} \mathrm{Te}_{4}$, i.e.

(GeTe):( $\left.\mathrm{Sb}_{2} \mathrm{Te}_{3}\right)=1: 1 ; \mathrm{Ge}_{2} \mathrm{Sb}_{2} \mathrm{Te}_{5}$, i.e. $(\mathrm{GeTe}):\left(\mathrm{Sb}_{2} \mathrm{Te}_{3}\right)=2: 1 ; \mathrm{Ge}_{4} \mathrm{Sb}_{2} \mathrm{Te}_{7}$, i.e.

(GeTe):( $\left.\mathrm{Sb}_{2} \mathrm{Te}_{3}\right)=4: 1 ; \mathrm{Ge}_{6} \mathrm{Sb}_{2} \mathrm{Te}_{9}$, i.e. $(\mathrm{GeTe}):\left(\mathrm{Sb}_{2} \mathrm{Te}_{3}\right)=6: 1$. In comparison with the nominal composition, the as-deposited films sputtered from single targets at RF power of $20 \mathrm{~W}$ are only slightly overstoichiometric in Ge or Sb content (+2.8 at. \% for Ge, +2.7 at. \% for Sb). For co-sputtered layers, broad range of composition was successfully covered ( 17.4-35.0 at. $\%$ of Ge, $14.2-29.0$ at. \% of Sb) with slight variation in Te content (50.8-53.6 at. \% of Te) by varying RF power on the two cathodes. We note that the error limit of the used EDS method is \pm 1 at. $\%$. 
Thin films fabricated by (co-)sputtering technique were homogeneous and amorphous as confirmed by optical/electron microscopy, except of sputtered $\mathrm{Sb}_{2} \mathrm{Te}_{3}$ films which were partially crystalline (traces of rhombohedral $\mathrm{Sb}_{2} \mathrm{Te}_{3}$ in $\mathrm{Sb}_{2} \mathrm{Te}_{3}$ films) as determined from XRD patterns. On annealing of sputtered GeTe and co-sputtered Ge-Sb-Te films at $230^{\circ} \mathrm{C}$, crystallization proceeds while cubic symmetry $(F m \overline{3} m)$ of the annealed films was confirmed by XRD data. The annealing of $\mathrm{Sb}_{2} \mathrm{Te}_{3}$ layers leads to the formation of rhombohedral $\mathrm{Sb}_{2} \mathrm{Te}_{3}$ with large preferential orientation.

The films’ morphology studied via SEM, depicted as inset of Fig. 1, showed smooth surface of deposited films without any significant cracks or holes. For all as-deposited thin films, root mean squared roughness values $(S q)$ determined by AFM (exemplified as inset in Fig. 1) were found to be lower than $\sim 0.3 \mathrm{~nm}$ confirming smooth surface of the layers. On the other hand, films’ roughness determined by AFM for annealed layers increased (up to 1.4 $\mathrm{nm}$ for GeTe films) which is consistent with the crystallization phenomenon of the films upon annealing.

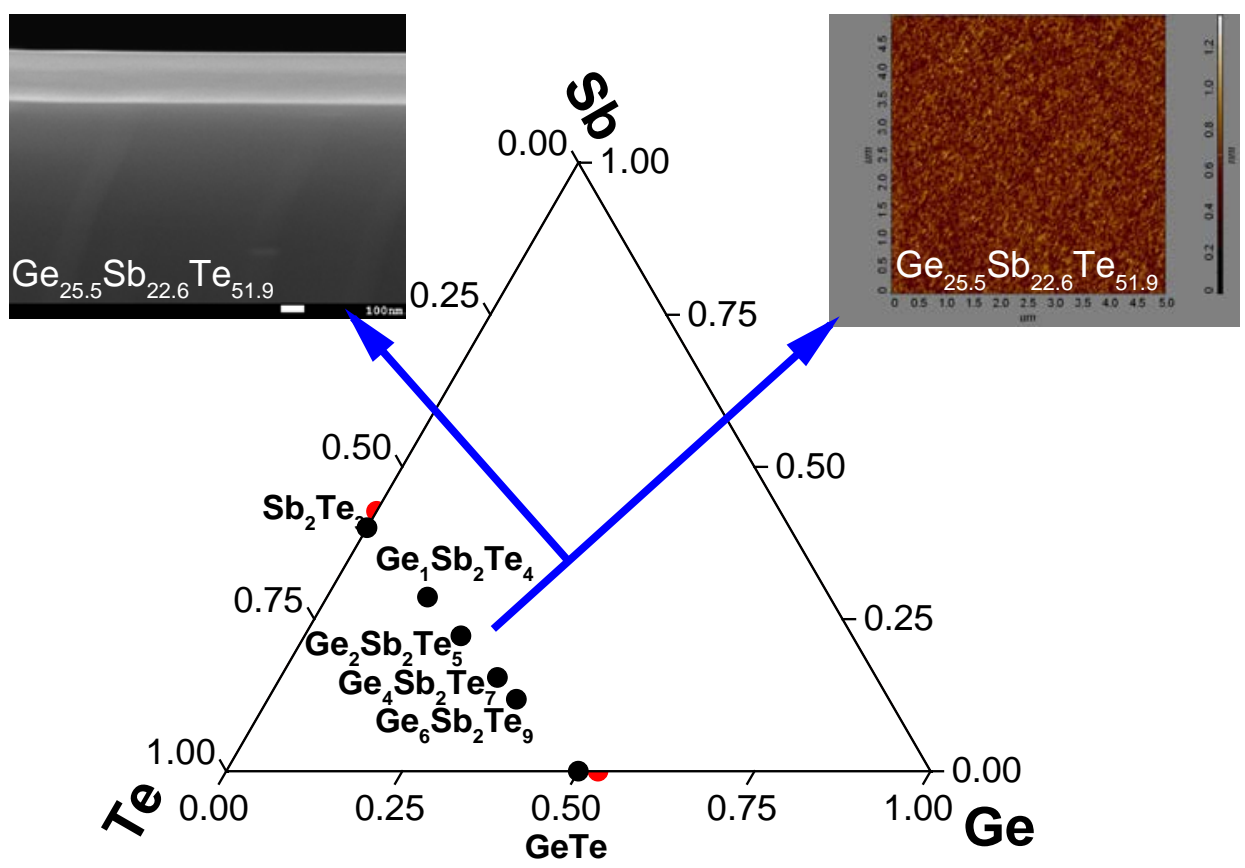

Fig. 1 Ternary Ge-Sb-Te diagram with indicated positions of (co-)sputtered thin films’ composition (red symbols), targets’ composition as well as selected stoichiometric 
compositions located on GeTe-Sb $2 \mathrm{Te}_{3}$ tie-line (black symbols). Right inset illustrates AFM scan $(5 \mu \mathrm{m} \times 5 \mu \mathrm{m})$ of $\mathrm{Ge}_{25.5} \mathrm{Sb}_{22.6} \mathrm{Te}_{51.9}$ thin film. Left inset shows SEM micrograph of crosssection of the same film.

Table 1. Characteristics of co-sputtered Ge-Sb-Te thin films: chemical composition (from EDS, \pm 1 at.\%), crystallization temperatures (amorphous $\rightarrow$ fcc, $T_{c 1}$; fcc $\rightarrow$ hexagonal, $T_{c 2} ; \pm 1$ ${ }^{\circ} \mathrm{C}$ ) and electrical contrast values (in terms of sheet resistance ratio between crystalline and amorphous phases $\left.R_{s(c r)} / R_{s(a m)}\right)$ determined from sheet resistance measurements, thickness ratio between crystalline and amorphous state $d_{c r} / d_{a m}$, optical band gap for both, crystalline and amorphous films $\left(E_{g}{ }^{\text {opt }}, \pm 0.02 \mathrm{eV}\right)$, optical contrast $\Delta n+i \Delta k$ (n,k(crystalline)$n, k$ (amorphous)) values at wavelength of $400 \mathrm{~nm}$, absolute values of optical contrast at 400 $\mathrm{nm}$ in terms of $|\Delta n|+|\Delta k|$, and dielectric function $\varepsilon_{\infty}$ for both, crystalline and amorphous layers, determined from spectroscopic ellipsometry. The films used for EDS measurements were deposited onto silicon substrates, while those for sheet resistance and spectroscopic ellipsometry measurements were prepared onto glass substrates. Note that error in determination of thickness is $\pm 2 \mathrm{~nm}$.

\begin{tabular}{|c|c|c|c|c|c|c|c|}
\hline $\begin{array}{l}\text { Chemical } \\
\text { composition }\end{array}$ & $\begin{array}{c}T_{C 1} \mid T_{C 2} \\
\left({ }^{\circ} \mathrm{C}\right)\end{array}$ & $R_{S(c r)} / R_{S(a m)}$ & $d_{c r} / d_{a m}$ & $\begin{array}{c}E_{g}^{o p t} \\
\mathrm{cr} \mid \mathrm{am} \\
(\mathrm{eV})\end{array}$ & $\begin{array}{c}\Delta n+i \Delta k \\
@ 400 \mathrm{~nm}\end{array}$ & $\begin{array}{l}|\Delta n|+|\Delta k| \\
\text { @ } 400 \mathrm{~nm}\end{array}$ & $\begin{array}{c}\varepsilon_{\infty} \\
\mathrm{cr} \mid \mathrm{am}\end{array}$ \\
\hline $\mathrm{Ge}_{52.8} \mathrm{Te}_{47.2}$ & $190 \mid-$ & $2.7 \times 10^{-8}$ & 0.845 & $0.45 \mid 0.66$ & $-1.20+i 1.76$ & 2.96 & $26.5 \mid 12.7$ \\
\hline $\mathrm{Ge}_{35.0} \mathrm{Sb}_{14.2} \mathrm{Te}_{50.8}$ & 169 | 295 & $8.0 \times 10^{-8}$ & 0.870 & $0.50 \mid 0.71$ & $-1.24+i 1.51$ & 2.75 & $34.2 \mid 13.6$ \\
\hline $\mathrm{Ge}_{30.5} \mathrm{Sb}_{17.6} \mathrm{Te}_{51.9}$ & $163 \mid 287$ & $1.1 \times 10^{-7}$ & 0.907 & $0.47 \mid 0.71$ & $-0.99+i 1.33$ & 2.32 & $34.3 \mid 14.1$ \\
\hline $\mathrm{Ge}_{25.5} \mathrm{Sb}_{22.6} \mathrm{Te}_{51.9}$ & $157 \mid 283$ & $1.8 \times 10^{-7}$ & 0.901 & $0.49 \mid 0.69$ & $-0.99+i 1.27$ & 2.26 & $33.8 \mid 14.7$ \\
\hline $\mathrm{Ge}_{21.9} \mathrm{Sb}_{24.9} \mathrm{Te}_{53.2}$ & $150 \mid 285$ & $9.9 \times 10^{-7}$ & 0.953 & $0.47 \mid 0.68$ & $-0.88+i 0.82$ & 1.70 & $32.1 \mid 15.3$ \\
\hline $\mathrm{Ge}_{17.4} \mathrm{Sb}_{29.0} \mathrm{Te}_{53.6}$ & 145 | 277 & $1.1 \times 10^{-6}$ & 0.935 & $0.38 \mid 0.66$ & $-0.73+i 0.88$ & 1.61 & $31.7 \mid 16.3$ \\
\hline $\mathrm{Sb}_{42.7} \mathrm{Te}_{57.3}$ & $-1-$ & $9.4 \times 10^{-3}$ & 1.00 & $0.29 \mid 0.45$ & $-0.23+i 0.52$ & 0.75 & $41.6 \mid 35.7$ \\
\hline
\end{tabular}


The crystallization temperatures were evaluated based on temperature dependences of thin films' sheet resistance $\left(R_{s}\right)$. The crystallization of the co-sputtered amorphous Ge-Sb-Te films into fcc phase was identified in the region of $\sim 145-169{ }^{\circ} \mathrm{C}$, while transition from fcc to hexagonal phase proceeds between 277 and $295^{\circ} \mathrm{C}$ (Table 1). The former one is characterized by abrupt decrease of $R_{S}$ (Fig. 2). Both transitions are more precisely determined as peak temperatures of $d\left(\ln R_{s}\right) / d(1 / T)$ first derivatives. The crystallization temperatures are slightly dependent on content of GeTe in the films. On the other hand, sputtered GeTe film shows only amorphous-to-fcc phase transition occurring at $\sim 190{ }^{\circ} \mathrm{C}$. For sputtered $\mathrm{Sb}_{2} \mathrm{Te}_{3}$ layer, the phase transition (amorphous-to-hexagonal) is not sudden but rather evidenced by continuous decrease of sheet resistance. Table 1 also summarizes electrical contrast values (in terms of sheet resistance ratio between crystalline and amorphous phases $\left.R_{s(c r)} / R_{s(a m)}\right)$ determined from sheet resistance measurements, showing broad range covering several orders of magnitude. With growing content of GeTe in co-sputtered Ge-Sb-Te thin films, the electrical contrast is increasing up to $R_{S(c r)} / R_{S(a m)} \sim 8.0 \times 10^{-8}$ for $\mathrm{Ge}_{35.0} \mathrm{Sb}_{14.2} \mathrm{Te}_{50.8}$, while the highest electrical contrast was observed for sputtered GeTe layer $\left(\sim 2.7 \times 10^{-8}\right)$.

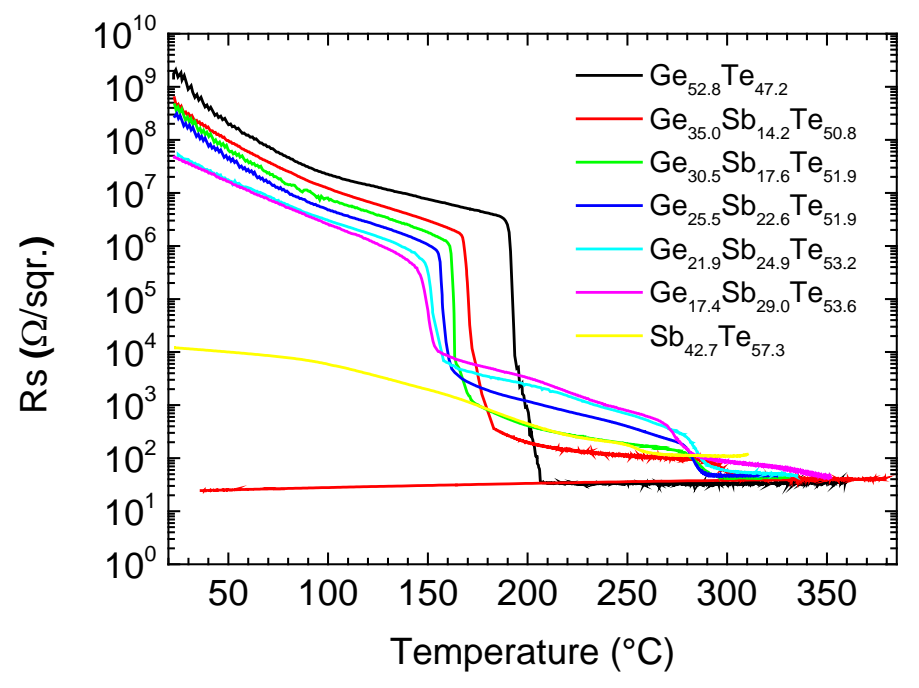

Fig. 2 Sheet resistance of as-deposited Ge-Sb-Te layers (deposited on microscope glass substrates) temperature dependence. 
The thickness of the as-deposited samples under study, determined by ellipsometry, was in the range of $\sim 110-180 \mathrm{~nm}$ (range enabling easy implementation of spectroscopic ellipsometry data analysis). On the other hand, the thickness of annealed layers (except $\mathrm{Sb}_{2} \mathrm{Te}_{3}$ ) was $~ 5-15 \%$ lower in comparison with the thicknesses of as-deposited ones (Table 1); observed thickness changes are comparable with that ones for Ge-Sb-Te thin films reported earlier ( 3-13\% for pulsed lased deposited layers $[14,15])$. The largest thickness decrease due to amorphous-to-fcc phase transition was detected for sputtered GeTe film.

Application of CL model for ellipsometry data analysis allowed the determination of band gap $\left(E_{g}\right)$ values for both as-deposited as well as fcc crystalline films (Table 1). Sputtered GeTe and co-sputtered Ge-Sb-Te films exhibit $E_{g}$ values in range of $0.66-0.71$ and $0.38-0.50$ $\mathrm{eV}$ for amorphous and crystalline thin films, respectively, correlating well with already published data [15]. Upon crystallization, the $E_{g}$ values of studied Ge-Sb-Te films decrease (about 0.2-0.3 eV) which is consistent with previously reported results [15, 25]. Band gap energy determined for sputtered as-deposited $\mathrm{Sb}_{2} \mathrm{Te}_{3}$ layer $(\sim 0.45 \mathrm{eV})$ is consistent with data found in the literature (optical bandgap of $\sim 0.52-0.80 \mathrm{eV}$ in amorphous state and bandgap energies of $\sim 0.15-0.32 \mathrm{eV}$ in crystalline state [15]) taking into account fact that the layer is partially crystalline.

Best fit optical functions, i.e. refractive index and extinction coefficient spectral dependences, deducted from spectroscopic ellipsometry data analysis for both, the asdeposited and annealed Ge-Sb-Te thin films are shown in Figs 3 and 4, respectively. As seen, in studied spectral and compositional region, the refractive index and extinction coefficient vary drastically. To assess the refractive index and extinction coefficient changes, optical contrast $\Delta n+i \Delta k$ ( $n, k$ (annealed)- $n, k($ as-deposited)) values at wavelength of $400 \mathrm{~nm}$ and absolute values of optical contrast at $400 \mathrm{~nm}$ in terms of $|\Delta n|+|\Delta k|$ were extracted and summarized in Table 1. Lowest values of optical contrast as well as its absolute value were 
found for binary $\mathrm{Sb}_{2} \mathrm{Te}_{3}$ layers $(\Delta n+i \Delta k=-0.23+\mathrm{i} 0.52,|\Delta n|+|\Delta k|=0.75)$. Contrary, cosputtered Ge-Sb-Te films show particularly larger optical contrast reaching values of $\Delta n+i \Delta k$ $=-1.24+\mathrm{i} 1.51$ and $|\Delta n|+|\Delta k|=2.75$ for $\mathrm{Ge}_{35.0} \mathrm{Sb}_{14.2} \mathrm{Te}_{50.8}$ composition. Highest values were observed for sputtered GeTe films $(\Delta n+i \Delta k=-1.20+\mathrm{i} 1.76,|\Delta n|+|\Delta k|=2.96)$. Optical contrast values achieved in this work are generally comparable with similar compositions of the films from Ge-Sb-Te system [8]. It is evident that larger content of GeTe in Ge-Sb-Te layers leads to higher value of optical contrast.
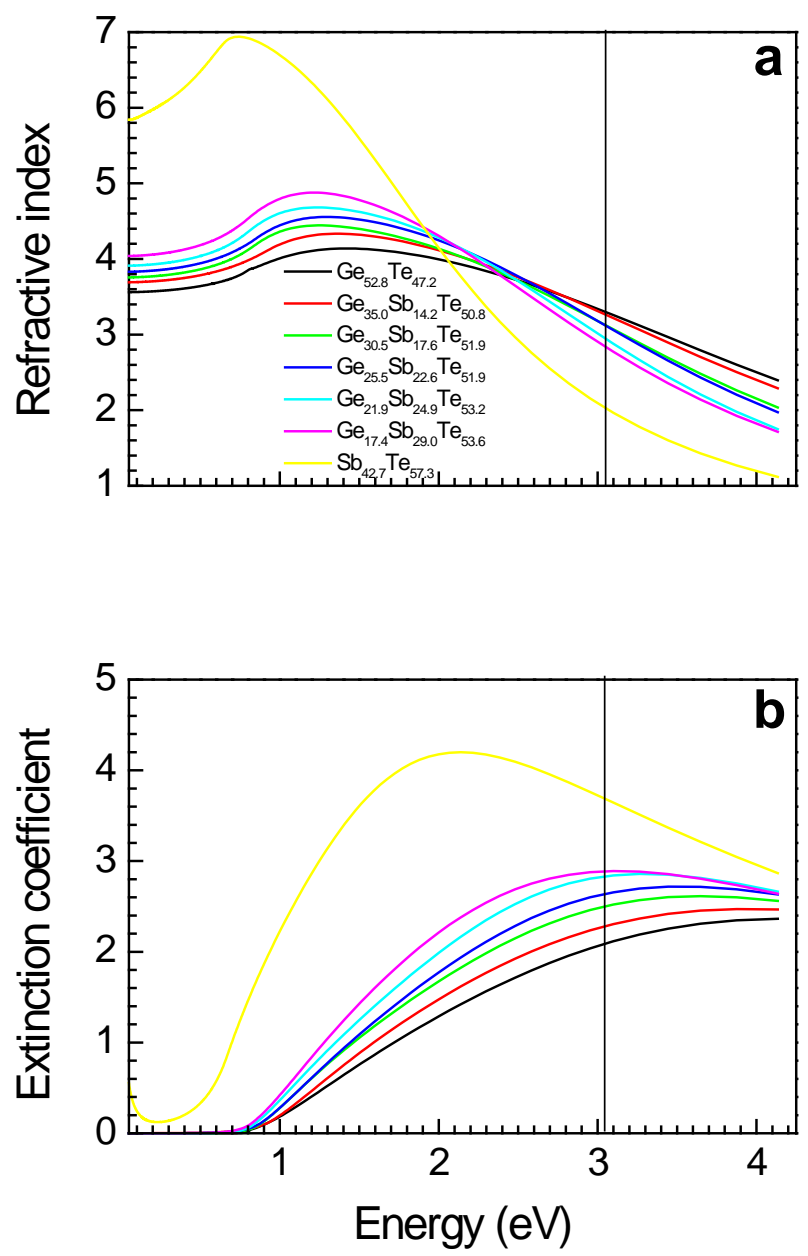

Fig. 3 Optical functions of as-deposited (co-)sputtered Ge-Sb-Te thin films deposited on microscope glass substrates: (a) spectral dependences of refractive indices, (b) spectral dependences of extinction coefficients. Vertical lines represent wavelength of $405 \mathrm{~nm}$ used in BD technology. 

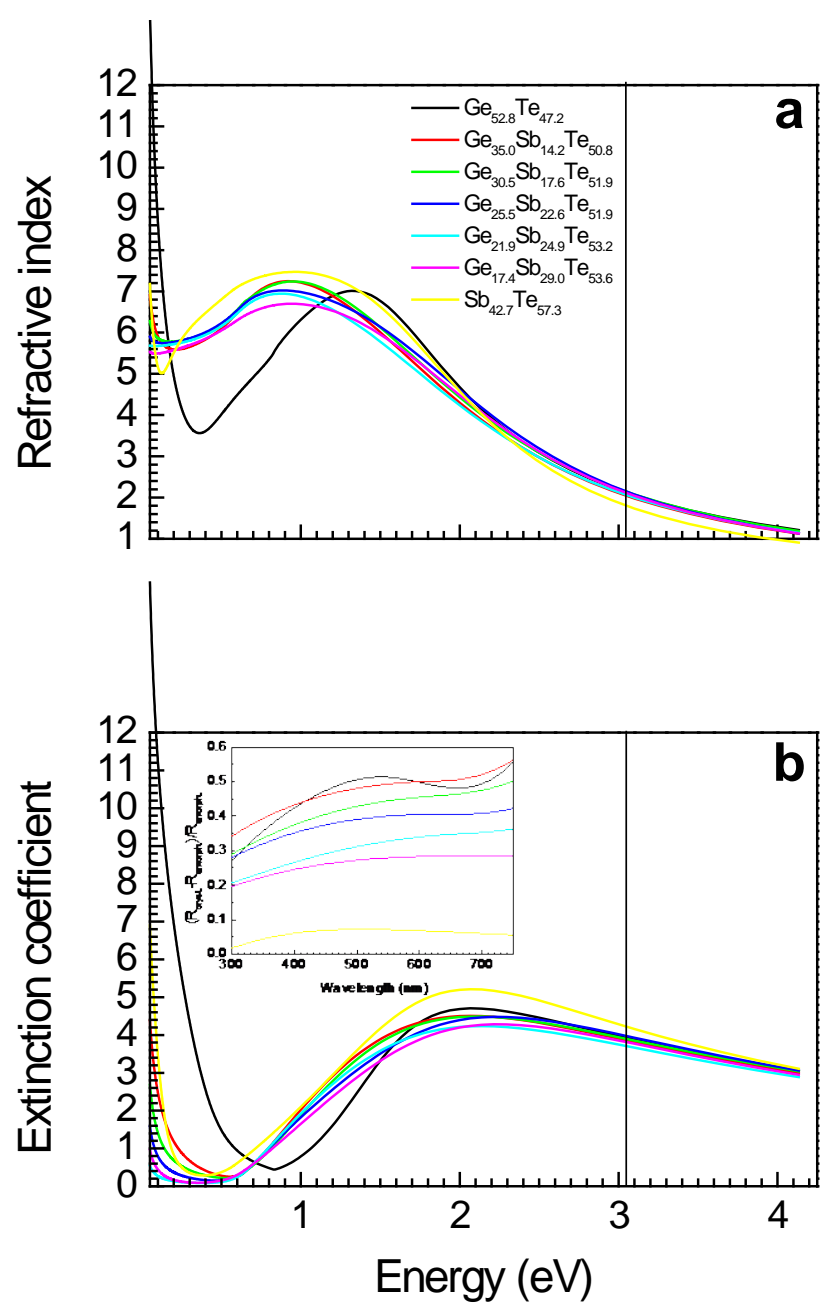

Fig. 4 Optical functions of annealed (co-)sputtered Ge-Sb-Te thin films deposited on microscope glass substrates: (a) spectral dependences of refractive indices, (b) spectral dependences of extinction coefficients. Inset shows calculated reflectivity contrast $\left(R_{c r^{-}}\right.$ $\left.R_{a m}\right) / R_{a m}$. Vertical lines represent wavelength of $405 \mathrm{~nm}$ used in BD technology.

The optical constant $\varepsilon_{\infty}$ was determined using dielectric function $\varepsilon_{1}$ as its value at 0.05 eV (energy close to zero) after subtraction of Drude contribution to the absorption where necessary (crystalline samples as well as as-deposited $\mathrm{Sb}_{2} \mathrm{Te}_{3}$ layers). The values of $\varepsilon_{\infty}$, listed in Table 1, present behaviour typical for phase change materials with lower ( 13-16) and larger ( 27-34) values for amorphous and crystalline phase, respectively. Note that high value of $\varepsilon_{\infty}$ for as-deposited $\mathrm{Sb}_{2} \mathrm{Te}_{3}$ films is because these films are partially crystalline. 
Using ellipsometry data, reflectivity spectral dependences of both, amorphous and crystalline Ge-Sb-Te films were also calculated and plotted as ratio $\left(R_{c r}-R_{a m}\right) / R_{a m}$ (inset of Fig. 4). Calculated reflectivity contrast at BD wavelength increases with increasing content of GeTe in Ge-Sb-Te thin films up to $43 \%$, confirming importance of GeTe content for this group of phase change memory materials.

\section{Conclusions}

In this work, Ge-Sb-Te amorphous thin films were fabricated by RF magnetron co-sputtering technique exploiting GeTe and $\mathrm{Sb}_{2} \mathrm{Te}_{3}$ targets. The exploited fabrication technique allowed depositing thin films within broad range of chemical composition ( 17.4-35.0 at. \% of Ge, $\sim 14.2-29.0$ at. \% of Sb) with slight variation in Te content (50.8-53.6 at. \% of Te). As seen from AFM and SEM observations, the quality of fabricated Ge-Sb-Te amorphous layers is good, with smooth surface, without significant cracks or holes. On annealing at $230{ }^{\circ} \mathrm{C}$, the amorphous Ge-Sb-Te films undergo phase change to crystalline layers with cubic symmetry $(F m \overline{3} m)$ as results from XRD patterns. Sheet resistance temperature dependences revealed that the electrical contrast between annealed and as-deposited films is increasing with growing content of GeTe in co-sputtered Ge-Sb-Te thin films up to $R_{s(c r)} / R_{s(a m)} \sim 2.7 \times 10^{-8}$ for GeTe layers. Phase transition from amorphous to crystalline state is accompanied by the particular thickness change of the films; its decreases on annealing by 5-15\% was observed. Optical band gap values (0.66-0.71 and 0.38-0.50 eV for amorphous and crystalline thin films, respectively) show their decrease of about 0.2-0.3 eV upon crystallization. Huge changes of optical functions are also demonstrated by optical contrast values up to $|\Delta n|+|\Delta k|$ = 2.96 for GeTe layers at Blu-ray wavelength. Finally, reflectivity contrast at this wavelength reaches up to $\sim 43 \%$ with increasing content of GeTe in Ge-Sb-Te thin films. All the obtained data confirm importance of GeTe content in Ge-Sb-Te thin films. Based on achieved results, 
co-sputtering was proven to be prospective method the fabrication of thin films with potential applications in the field of phase change memory materials.

\section{Acknowledgements}

Support from the Czech Science Foundation (Project No. 18-03823S) is greatly

acknowledged. The authors appreciate also the financial support from LM2018103 project funded by the Ministry of Education, Youth and Sports of the Czech Republic. Authors are thankful to Assoc. Prof. L. Beneš and Mr. J. Přikryl for XRD and sheet resistance temperature dependences measurements, respectively.

Competing financial interests: The authors declare no competing financial interests.

Author contribution statement: Conceptualization, P.N. and V.N.; methodology, V.N. and P.N.; investigation, M.B., J.G., T.H., V.N. and P.N.; writing—original draft preparation, P.N.; writing - review and editing, P.N. and V.N.; funding acquisition, P.N. 


\section{References}

[1] S.R. Ovshinsky, Reversible Electrical Switching Phenomena in Disordered Structures, Phys. Rev. Lett. 21 (1968) 1450-1453.

[2] N. Yamada, E. Ohno, K. Nishiuchi, N. Akahira, M. Takao, Rapid-phase transitions of GeTe- $\mathrm{Sb}_{2} \mathrm{Te}_{3}$ pseudobinary amorphous thin films for an optical disk memory, J. Appl. Phys. 69 (1991) 2849-2856. [3] H. Iwasaki, Y. Ide, M. Harigaya, Y. Kageyama, I. Fujimura, Completely erasable phase-change optical disk, Japan. J. Appl. Phys. Part 1-Regular Papers Short Notes \& Review Papers 31 (1992) 461 465.

[4] S. Raoux, W. Welnic, D. Ielmini, Phase change materials and their application to nonvolatile memories, Chem. Rev. 110 (2010) 240-267.

[5] I. Friedrich, V. Weidenhof, W. Njoroge, P. Franz, M. Wuttig, Structural transformations of $\mathrm{Ge}_{2} \mathrm{Sb}_{2} \mathrm{Te}_{5}$ films studied by electrical resistance measurements, J. Appl. Phys. 87 (2000) 4130-4134.

[6] W. Welnic, M. Wuttig, Reversible switching in phase-change materials, Mater. Today 11 (2008) 20-27.

[7] M. Wuttig, N. Yamada, Phase-change materials for rewriteable data storage, Nat. Mater. 6 (2007) 824-832.

[8] N. Yamada, Origin, secret, and application of the ideal phase-change material GeSbTe, Phys. Status Solidi B-Basic Solid State Phys. 249 (2012) 1837-1842.

[9] W. Zhang, R. Mazzarello, M. Wuttig, E. Ma, Designing crystallization in phase-change materials for universal memory and neuro-inspired computing, Nat. Rev. Mater. 4 (2019) 150-168.

[10] S. Raoux, M. Wuttig, Phase Change Materials, Springer, New York, 2009.

[11] M. Wuttig, H. Bhaskaran, T. Taubner, Phase-change materials for non-volatile photonic applications, Nature Photon. 11 (2017) 465-476.

[12] E. Garcia-Garcia, A. Mendoza-Galvan, Y. Vorobiev, E. Morales-Sanchez, J. Gonzalez-Hernandez, G. Martinez, B.S. Chao, Optical properties of Ge : Sb : Te ternary alloys, J. Vac. Sci. Technol. A-Vacuum Surfaces and Films 17 (1999) 1805-1810.

[13] J. Orava, T. Wagner, J. Sik, J. Prikryl, M. Frumar, L. Benes, Optical properties and phase change transition in $\mathrm{Ge}_{2} \mathrm{Sb}_{2} \mathrm{Te}_{5}$ flash evaporated thin films studied by temperature dependent spectroscopic ellipsometry, J. Appl. Phys. 104 (2008) 043523.

[14] M. Bouska, S. Pechev, Q. Simon, R. Boidin, V. Nazabal, J. Gutwirth, E. Baudet, P. Nemec, Pulsed laser deposited GeTe-rich GeTe-Sb $2 \mathrm{Te}_{3}$ thin films, Sci. Rep. 6 (2016) 26552.

[15] P. Nemec, J. Prikryl, V. Nazabal, M. Frumar, Optical characteristics of pulsed laser deposited GeSb-Te thin films studied by spectroscopic ellipsometry, J. Appl. Phys. 109 (2011) 073520.

[16] A. Abrutis, V. Plausinaitiene, M. Skapas, C. Wiemer, O. Salicio, A. Pirovano, E. Varesi, S.

Rushworth, W. Gawelda, J. Siegel, Hot-wire chemical vapor deposition of chalcogenide materials for phase change memory applications, Chem. Mat. 20 (2008) 3557-3559.

[17] B.J. Choi, S. Choi, Y.C. Shin, C.S. Hwang, J.W. Lee, J. Jeong, Y.J. Kim, S.Y. Hwang, S.K. Hong, Cyclic PECVD of $\mathrm{Ge}_{2} \mathrm{Sb}_{2} \mathrm{Te}_{5}$ films using metallorganic sources, J. Electrochem. Soc. 154 (2007) H318-H324.

[18] M. Ritala, V. Pore, T. Hatanpaa, M. Heikkila, M. Leskela, K. Mizohata, A. Schrott, S. Raoux, S.M. Rossnagel, Atomic layer deposition of $\mathrm{Ge}_{2} \mathrm{Sb}_{2} \mathrm{Te}_{5}$ thin films, Microelectron. Eng. 86 (2009) 1946-1949. [19] V. Nazabal, P. Nemec, Amorphous Thin Film Deposition, in: J.D. Musgraves, J. Hu, L. Calvez (Eds.) Springer Handbook of Glass, Springer International Publishing, Cham, 2019, pp. 1291-1330.

[20] E. Baudet, M. Sergent, P. Nemec, C. Cardinaud, E. Rinnert, K. Michel, L. Jouany, B. Bureau, V. Nazabal, Experimental design approach for deposition optimization of RF sputtered chalcogenide thin films devoted to environmental optical sensors, Sci. Rep. 7 (2017) 3500.

[21] T. Halenkovic, J. Gutwirth, P. Nemec, E. Baudet, M. Specht, Y. Gueguen, J.C. Sangleboeuf, V. Nazabal, Amorphous Ge-Sb-Se thin films fabricated by co-sputtering: Properties and photosensitivity, 
J. Am. Ceram. Soc. 101 (2018) 2877-2887.

[22] T. Halenkovič, J. Gutwirth, M. Bouška, L. Calvez, P. Němec, V. Nazabal, Amorphous Ga-Sb-Se thin films fabricated by co-sputtering, Opt. Lett. 45 (2020) 29-32.

[23] A.V. Kolobov, P. Fons, A.I. Frenkel, A.L. Ankudinov, J. Tominaga, T. Uruga, Understanding the phase-change mechanism of rewritable optical media, Nat. Mater. 3 (2004) 703-708.

[24] J.W. Park, S.H. Baek, T.D. Kang, H. Lee, Y.S. Kang, T.Y. Lee, D.S. Suh, K.J. Kim, C.K. Kim, Y.H. Khang, J.L.F. Da Silva, S.H. Wei, Optical properties of $\left(\mathrm{GeTe}, \mathrm{Sb}_{2} \mathrm{Te}_{3}\right)$ pseudobinary thin films studied with spectroscopic ellipsometry, Appl. Phys. Lett. 93 (2008) 021914.

[25] B.S. Lee, J.R. Abelson, S.G. Bishop, D.H. Kang, B.K. Cheong, K.B. Kim, Investigation of the optical and electronic properties of $\mathrm{Ge}_{2} \mathrm{Sb}_{2} \mathrm{Te}_{5}$ phase change material in its amorphous, cubic, and hexagonal phases, J. Appl. Phys. 97 (2005) 093509.

[26] J.Y. Cho, D. Kim, Y.J. Park, T.Y. Yang, Y.Y. Lee, Y.C. Joo, The phase-change kinetics of amorphous $\mathrm{Ge}_{2} \mathrm{Sb}_{2} \mathrm{Te}_{5}$ and device characteristics investigated by thin-film mechanics, Acta Mater. 94 (2015) 143151.

[27] S.J. Wei, H.F. Zhu, K. Chen, D. Xu, J. Li, F.X. Gan, X. Zhang, Y.J. Xia, G.H. Li, Phase change behavior in titanium-doped $\mathrm{Ge}_{2} \mathrm{Sb}_{2} \mathrm{Te}_{5}$ films, Appl. Phys. Lett. 98 (2011) 231910.

[28] Y.Q. Zhu, Z.H. Zhang, S.N. Song, H.Q. Xie, Z.T. Song, X.Y. Li, L.L. Shen, L. Li, L.C. Wu, B. Liu, Nidoped GST materials for high speed phase change memory applications, Mater. Res. Bull. 64 (2015) 333-336.

[29] Q. Gao, L. Chen, Effect of Cu doping on microstructure and thermal stability of $\mathrm{Ge}_{2} \mathrm{Sb}_{2} \mathrm{Te}_{5}$ thin film, Appl. Phys. A-Mater. Sci. Process. 125 (2019) 564.

[30] S. Buller, C. Koch, W. Bensch, P. Zalden, R. Sittner, S. Kremers, M. Wuttig, U. Schuermann, L. Kienle, T. Leichtweiss, J. Janek, B. Schoenborn, Influence of Partial Substitution of Te by Se and Ge by $\mathrm{Sn}$ on the Properties of the Blu-ray Phase-Change Material $\mathrm{Ge}_{8} \mathrm{Sb}_{2} \mathrm{Te}_{11}$, Chem. Mat. 24 (2012) 35823590.

[31] Z.G. Li, Y.G. Lu, Y.D. Ma, S.N. Song, X. Shen, G.X. Wang, S.X. Dai, Z.T. Song, Changes in electrical and structural properties of phase-change Ge-Sb-Te films by Zr addition, J. Non-Cryst. Solids 452 (2016) 9-13.

[32] T.J. Park, S.Y. Choi, M.J. Kang, Phase transition characteristics of $\mathrm{Bi} / \mathrm{Sn}$ doped $\mathrm{Ge}_{2} \mathrm{Sb}_{2} \mathrm{Te}_{5}$ thin film for PRAM application, Thin Solid Films 515 (2007) 5049-5053.

[33] R. Mawale, T. Halenkovic, M. Bouska, J. Gutwirth, V. Nazabal, P.L. Bora, L. Pecinka, L. Prokes, J. Havel, P. Nemec, Mass spectrometric investigation of amorphous Ga-Sb-Se thin films, Sci. Rep. 9 (2019) 10213.

[34] L.J. Van der Pauw, A method of measuring specific resistivity and Hall effect of discs of arbitrary shape, Philips Research Reports 13 (1958) 1-9.

[35] G.D. Cody, in: J.I. Pankove (Ed.) Semiconductors and semimetals, Academic, Orlando, FL, 1984, pp. 11.

[36] E. Baudet, A. Gutierrez-Arroyo, P. Nemec, L. Bodiou, J. Lemaitre, O. De Sagazan, H. Lhermitte, E. Rinnert, K. Michel, B. Bureau, J. Charrier, V. Nazabal, Selenide sputtered films development for MIR environmental sensor, Opt. Mater. Express 6 (2016) 2616-2627. 